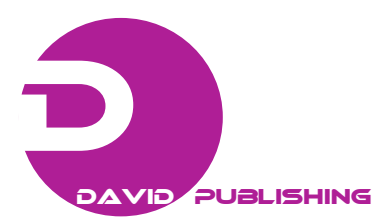

\title{
Impact of Vulnerable Group Development Program on Food Security in Kushtia Sadar Upazila of Bangladesh
}

\author{
Md. Safiul Islam Afrad ${ }^{*}$, Shahoriar Ferdous ${ }^{2}$, Md. Abdul Muttaleb ${ }^{3}$, Md. Mizanur Rahman ${ }^{4}$ \\ 1. Bangabandhu Sheikh Mujibur Rahman Agricultural University (BSMRAU), Gazipur \\ 2. Department of Agricultural Extension and Rural Development, Bangladesh Agricultural Research Institute, Gazipur \\ 3. Bangladesh Rice Research Institute, Gazipur \\ 4. Dept of Soil Science, BSMRAU, Gazipur-1706, Bangladesh \\ *Corresponding author's e-mail: afrad69@gmail.com
}

\begin{abstract}
The main objectives guided the present study are to assess the socio-demographic characteristics of the beneficiaries' of vulnerable group development (VGD) program and its impact on the beneficiaries along with related factors. The study was conducted in sadar upazila of Kushtia district in Bangladesh. Data were collected from 70 selected beneficiaries of VGD program by employing simple random sampling technique using semi structured and pre-tested interview schedule. Data revealed that majority of the beneficiary were middle aged (average 33.36 years) with poor education (average 2.21 years), medium family size (average 4.97), day labor (70\%), very poor monthly family income (61.40\% less than 2000.00), Muslim (81.40\%), politically affiliated (88.60\%) and involved with NGOs (58.60\%). Due to the involvement of VGD program, overwhelming majority (91.40\%) of the beneficiaries stated that amount of food support was sufficient for 15-20 days of a month that enhanced $15.24 \%$ more day's food security. On the other hand, number of meal per day increased 32.48\% after being involved with the VGD program. However, food security and number of meal per day increased significantly with the involvement of VGD program. Poor quality of supplied food (77\%), politically biased beneficiary selection system (77\%) and underweight (77\%) of delivered food were the problem as stated by the beneficiaries. The main problems mentioned by the beneficiaries were low grain quality (80\%), political biasness in selecting beneficiaries (75\%). Suggestions raised from the beneficiaries were improving the quality of grain (85\%) and selecting the actual persons without any bias (65\%).
\end{abstract}

Key words: Food security, impact, vulnerable group development, Kushtia, Bangladesh. 